Ger J Exerc Sport Res 2020 · 50:501-510 https://doi.org/10.1007/s12662-020-00673-8 Eingegangen: 13. Mai 2020

Angenommen: 29. Juli 2020

Online publiziert: 23. September 2020

(c) Der/die Autor(en) 2020

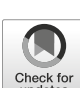

Jonas Wibowo

Bergische Universität Wuppertal, Wuppertal, Deutschland

\title{
Adaptivität im Sportunterricht in selbstständigen Arbeitsphasen messen
}

\section{Pilotierung eines Beobachtungsinstruments}

Der Begriff Adaptivität wird sehr vielfältig und bereits seit vielen Jahrzehnten in der Wissenschaft verwendet. Neben den sozialkonstruktivistischen Grundlagen ist den Beiträgen zu dem Thema gemeinsam, dass Adaptivität als ein wissenschaftlich fundierter Ansatz zum Umgang mit der Heterogenität von Lerngruppen gesehen wird (Corno \& Snow, 1986; Hardy, Decristan, \& Klieme, 2019; Helmke \& Weinert, 1997; Parsons et al., 2018). Als kleinster gemeinsamer Nenner einer Bestimmung von Adaptivität kann ein Verständnis dienen, das Adaptivität als eine Passung (Contingency) zwischen Lernvoraussetzungen und Nutzungsprozessen der Schüler*innen und den Instruktionen durch die Lehrperson ausdrückt. Je nach Forschungsansatz werden dabei die Instruktion der Lehrperson, die Lernprozesse der Schüler*innen oder die Passung selbst in den Mittelpunkt gerückt. In dem vorliegenden Beitrag wird ein Ansatz vorgestellt, der die Passung zwischen Instruktion der Lehrperson und den Lernaktivitäten der Schüler*innen fokussiert und versucht, dieses Verhältnis zwischen Lernen und Instruktion über ein standardisiertes Beobachtungsinstrument zu erfassen. Um begriffliche Missverständnisse mit einem allgemeinen oder auf Lehrpersonen gerichteten Verständnis von Passung bzw. Adaptivität zu vermeiden, wird für diese Verhältnisperspektive im Folgenden der Begriff Contingency verwendet.

\section{Contingency als Perspektive auf Adaptivität}

Hardy, Decristan, und Klieme (2019) unterscheiden in ihrem Überblicksbeitrag zum Thema Adaptivität zunächst eine Makro-Ebene (Unterrichtsplanung) und eine Mikro-Ebene (Handeln im laufenden Unterricht; s. auch Beck, Baer, Guldimann, Bischoff, \& Brühwiler, 2008; Corno \& Snow, 1986). Aufgrund der Ausrichtung des eigenen Ansatzes, wird im Folgenden vorwiegend auf die Mikro-Ebene eingegangen. Weiterhin unterscheiden Hardy et al. (2019) auf der MikroEbene unterrichtlichen Handelns eine Instruktions- und eine Verhältnisperspektive auf das Thema Adaptivität.

In der Instruktionsperspektive wird Adaptivität als Merkmal effektiven Unterrichtens verstanden. Gemeinsam ist den Ansätzen der Instruktionsperspektive, dass auf einer empirischen Ebene vornehmlich auf die Handlungen der Lehrperson fokussiert wird. Aus einzelnen Lehrpersonenhandlungen wird auf eine Passung bzw. das Potenzial $\mathrm{zu}$ einer Passung zu den Voraussetzungen oder Nutzungsprozessen der Schüler*innen geschlossen. Weiterhin wird von der Lehrpersonenhandlung angenommen, dass diese per se lernförderlich sei (Decristan et al., 2015). Diese Perspektive wird u.a. dafür kritisiert, dass individuelle Unterschiede der Schüler*innen nicht (ausreichend) berücksichtigt werden (Corno, 2008). Dem versucht die Verhältnisperspektive durch eine kontextualisierte Betrachtung der Instruktion der Lehrperson zu begegnen bzw. die Relation zwischen Instruktion und Voraussetzungen der Lernenden zu erfassen.

Der Verhältnisperspektive auf das Thema Adaptivität liegt die Annahme zugrunde, dass nicht eine konkrete Ausprägung des Handelns der Lehrperson pauschal als passend angenommen werden kann, sondern unterschiedliche Handlungen im Kontext unterschiedlicher Voraussetzungen und Nutzungsprozesse der Schüler*innen als lernförderlich oder auch nichtförderlich anzusehen sind (Corno, 2008; Van de Pol, Volman, \& Beishuizen, 2010). Anders als in der Instruktionsperspektive kann Passung daher nicht ausschließlich über Handlungen der Lehrpersonen erfasst werden, sondern muss im Kontext der Nutzungsprozesse der Schüler ${ }^{*}$ innen erfasst werden. Dieser Fokus auf die Relation zwischen Lernaktivitäten und Instruktion wird im Folgenden als Contingency-Perspektive bezeichnet.

Die Contingency-Perspektive wird vor allem mit dem Ansatz des Scaffoldings in Verbindung gebracht (Van de Pol et al., 2010; Wood, Bruner, \& Ross, 1976; im Sportunterricht Farias, Hastie, \& Mesquita, 2018; Wibowo, Bähr \& Gröben, 2014). Der Bestimmung von (mehr oder weniger) Contingency auf der Basis konkreter Angebots-Nutzungs-Konstellationen kommt in diesem Ansatz eine entscheidende Rolle zu. Ein theoretisch etabliertes und empirisch mehrfach verwendetes Prinzip (Hermkes, Mach, \& Minnameier, 2018; Van de Pol \& Elbers, 2013; Wischgoll, Pauli, \& Reusser, 2015) zur Bestimmung solcher Passungsver- 
hältnisse ist der Contingency-Approach (Wood, Wood, \& Middleton, 1978). Demnach sollen die Schüler ${ }^{*}$ innen in der individuellen "region of sensitivity“ (Wood et al., 1978, S. 133) aktiviert werden, die in Bezug auf die Anforderungen immer eine Stufe über dem aktuellen Können der Schüler*innen liegt, damit sie weder über- oder unterfordert werden.

\section{Forschungsstand zu Contingency auf der Mikro-Ebene}

Contingency ist bisher nur in wenigen Arbeiten mit standardisierten Instrumenten erfasst worden. Hardy et al. (2019) berücksichtigen in ihrem Überblicksbeitrag über adaptiven Unterricht zum Thema Contingency lediglich fünf Studien. Der größere Teil der Befunde stammt aus Studien mit qualitativen Designs, die wegen Platzgründen und aufgrund der forschungsmethodischen Ausrichtung der vorliegenden Studie nur eingeschränkt berücksichtigt werden.

In Bezug auf den Umfang von Contingency stellen Ayvazo und Ward (2011) aufgrund der Analyse des Unterrichts von zwei Sportlehrpersonen fest, dass Contingency in Unterrichtseinheiten, in denen die Lehrpersonen mit dem Thema besser vertraut sind höher ausgeprägt ist als in Unterrichtseinheiten, in denen die Lehrpersonen weniger gut mit dem Thema vertraut sind $(68-73 \%$ als „contingent“ eingestufte Schüler*innenLehrperson-Schüler*innen-Analyseeinheiten an den Gesamtinteraktionen bei vertrauten Themen; $40-49 \%$ als „contingent" eingestufte Analyseeinheiten bei weniger vertrauten Themen). Dies könnte auch als Hinweis darauf gedeutet werden, dass eine steigende Expertise bzw. eine höhere Vertrautheit mit einer Vielzahl an Themen durch eine höhere Expertise $\mathrm{zu}$ einem höheren durchschnittlichen Contingency-Wert führt. Bei der Untersuchung von Contingency im Sozialkundeunterricht von 30 Lehrpersonen beobachten Van de Pol, Volman, Oort, und Beishuizen (2015) im Rahmen der Evaluation einer Intervention zum Thema Scaffolding deutliche Zuwächse der Contingency-Werte nach der Intervention (MW: 0,42-0,43 in einer ungeschulten Vergleichsgruppe; MW: 0,87 nach einer Intervention in der Interventionsgruppe; es handelt sich um Mittelwerte der als „contingent“ (=1) und „nicht-contingent" (=0) eingestuften Lehrperson-Schüler*innenLehrperson-Analyseeinheiten).

Bezüglich der Wirkungen unterschiedlicher Contingency-Werte auf Nutzungsprozesse und Lernerfolge der Schüler*innen verweisen die Befunde darauf, dass hohe Contingency-Werte nicht immer mit einem höheren Lernerfolg oder schnellerem Lernfortschritt einhergehen, wie dies in der Fachliteratur teilweise angenommen wird (Hardy et al., 2019). Wischgoll et al. (2015) stellen fest, dass Contingency dann mit erfolgreichem Lernen zusammenhängt, wenn die Lehrperson instruiert und die Schüler*innen diesen Anweisungen folgen. Unterrichtssituationen mit niedrigerer Kontrolle der Lehrpersonen werden, auch wenn sie als „contingent" kodiert werden, vermehrt in solchen Lernsituationen festgestellt, die als nichterfolgreich in Bezug auf den Lernfortschritt der Schüler ${ }^{\star}$ innen eingestuft werden. Van de Pol und Elbers (2013) wiederum stellen fest, dass hohe Contingency-Werte dann mit Lernfortschritt zusammenhängen, wenn das initiale Verstehensniveau der Lernenden als niedrig eingestuft wird. Beide Studien verweisen darauf, dass einerseits Lehrpersonen mit unterschiedlichen Voraussetzungen der Schüler*innen unterschiedlich gut umgehen können und andererseits Contingency sich unterschiedlich auf Schüler*innen mit verschiedenen Voraussetzungen auswirkt. Auch die Studie von Van de Pol, Mercer, und Volman (2018) verweist darauf, dass hohe Contingency-Werte nicht unmittelbar $\mathrm{zu}$ Lernfortschritt führen, sondern nur dann, wenn die Schüler*innen die Impulse der Lehrperson auch tatsächlich aufgreifen.

Zusammenhänge zwischen Bestandteilen professioneller Kompetenzen und Adaptivität werden zwar in Übersichtsbeiträgen $z u$ dem Thema angenommen (Gräsel, Decristan, \& König, 2017; Hardy et al., 2019; Parsons et al., 2018), jedoch sind diese Zusammenhänge bisher nicht aus einer Contingency-Perspektive über- prüft worden. Besonders der diagnostischen Fähigkeit der Lehrperson wird eine hohe Bedeutung beigemessen, da davon ausgegangen wird, dass Lehrpersonen zuerst die Lernsituation der Schüler*innen erfassen müssen, um entsprechend darauf zu reagieren Gräsel et al. (2017). Das Ergebnis von Ayvazo und Ward (2011), das auf die Bedeutung der Vertrautheit der Lehrperson mit dem Unterrichtsinhalt für die Contingency-Werte verweist, kann durch Befunde der kompetenzorientierten Professionsforschung zur hohen Bedeutsamkeit des Fachdidaktischen Wissens für die Unterrichtsqualität und Lernleistung der Schüler ${ }^{*}$ innen gestützt werden (Kunter et al., 2013; im Sport: Heemsoth \& Wibowo, 2020; Vogler, Messmer \& Allemann, 2017; Wibowo \& Heemsoth, 2019).

Bezüglich der forschungsmethodischen Vorgehensweisen zur Erfassung von Contingency kann festgehalten werden, dass sich die vorhandenen Ansätze zur standardisierten Erfassung von Contingency deutlich unterscheiden, insbesondere hinsichtlich der Wahl der sog. Contingency-Einheiten und der Contingency-Regeln zur Einstufung der Analyseeinheiten als mehr der weniger passend bzw. „contingent“. Beispielsweise wählen Van de Pol und Elbers (2013) Contingency-Einheiten, die sich aus Lehrperson-Schüler*innen-LehrpersonTriplets zusammensetzen (Ayvazo \& Ward, 2011), verwenden Schüler*innenLehrperson-Schüler*innen-Triplets, und Hermkes et al. (2018) verwenden als Analyseeinheiten ganze Interaktionssequenzen, die sich von Ankunft bis zum Verlassen der Lehrperson bei der Beratung einer Gruppe erstrecken. Auch bezüglich der Contingency-Regeln zeigen sich deutliche Unterschiede. Zum Beispiel wählen Van de Pol, Volman, Elbers, und Beishuizen (2012) eine Relationierung von Verstehen (der Schüler*innen; drei Stufen) zur Kontrolle (durch die Lehrperson; fünf Stufen) und stützen sich bei der Operationalisierung der Contingency-Regeln auf die Regel, dass nach einem geringen Verstehen der Schüler*innen, eine höhere Kontrolle erfolgen soll als zuvor und bei einem niedrigen Verstehen der Schüler*innen eine niedrigere Kontrolle erfolgen soll als zuvor. Anders 
operationalisieren Wischgoll et al. (2015) den Contingency-Approach von Wood et al. (1978) durch die kognitive Aktivität der Schülerinnen (Inaktivität [niedrig], Reaktion, Ko-Konstruktion, Autonomie [hoch]) und die kognitive Aktivierung durch die Lehrperson (keine Aktivierung [niedrig], Instruktion, Ko-Konstruktion, Beobachtung [hoch]). Als "contingent" werden dann (ohne nähere Erläuterung) die Kombinationen InstruktionReaktion, Ko-Konstruktion-Ko-Konstruktion und Beobachtung-Autonomie eingestuft. Ein weiterer Grund für die eingeschränkte Vergleichbarkeit der verschiedenen Ansätze zu Contingency liegt in der inhaltlichen Ausrichtung. Arbeiten, die sich auf den Contingency-Approach von Wood et al. (1978) beziehen, fokussieren vor allem die $\mathrm{Au}$ tonomie der Schüler*innen, es lassen sich aber auch Schwerpunktsetzungen auf die unterrichtlichen Inhalte (Ayvazo \& Ward, 2011) oder emotionale Aspekte finden (Krammer, 2009). Angesichts der Inhaltsbezogenheit der ContingencyRegeln erscheint es sinnvoll und notwendig, diese mit Bezug auf fachliche Ansätze zu begründen und zu konzipieren. Die unterschiedlichen inhaltlichen Schwerpunktsetzungen werden im Folgenden als Contingency-Dimensionen bezeichnet (in Anlehnung an Corno \& Snow, 1986).

Die starken Unterschiede hinsichtlich der Konstruktion der ContingencyEinheiten, der Contingency-Regeln und die Unterschiede in den ContingencyDimensionen verdeutlichen die Notwendigkeit, diese Aspekte bei der Konzeptualisierung explizit herauszuarbeiten und im Sinne einer inhaltlichen Validität $\mathrm{zu}$ begründen (Wibowo \& Dyson, 2020, im Review).

\section{Konzeptualisierung von Contingency im Sportunterricht}

Angesichts der Vielzahl an vorgeschlagenen Dimensionen für Adaptivität (Hardy et al., 2019; Corno, 2008) wurden aus verschiedenen Gründen die Contingency-Dimensionen der Autonomie und des Problemlösens fokussiert. Beide Aspekte wurden durch eine vorangegangene qualitative Studie für den Sportunter-

Ger J Exerc Sport Res 2020 · 50:501-510 https://doi.org/10.1007/s12662-020-00673-8

(c) Der/die Autor(en) 2020

\section{J. Wibowo \\ Adaptivität im Sportunterricht in selbstständigen Arbeitsphasen messen. Pilotierung eines Beobachtungsinstruments}

\section{Zusammenfassung}

Adaptives Unterrichten wird als ein vielversprechender Ansatz gesehen, um mit der großen interindividuellen Vielfalt unter den Schüler*innen im Sportunterricht umzugehen. Die Bedeutung der Thematik ergibt sich einerseits aus der gleichstellungspolitischen Forderung einer individuellen Förderung, aber auch aus einer lern- und bildungstheoretischen Notwendigkeit, Lernumgebungen an individuelle Bedürfnisse anzupassen, um Lern- und Bildungsprozesse zu unterstützen. Dabei gilt die Passung zwischen der Instruktion der Lehrperson und den Voraussetzungen der Schüler*innen als zentrales Gütekriterium für den Umgang mit Heterogenität. Die Forschungslandschaft zu der Thematik ist durch eine enorme Vielfalt in Bezug auf die Auslegung von und den forschungsmethodischen Zugang zu adaptivem Unterrichten geprägt.
In dem Beitrag werden ein Ansatz zur standardisierten Beobachtung der Passung zwischen der lehrpersonseitigen Instruktion und den Lernaktivitäten der Schüler*innen sowie Ergebnisse einer Pilotierungsstudie vorgestellt. Passung (Contingency) wird dabei als Verhältnis von Instruktionen zu vorangegangen Lernaktivitäten operationalisiert. Die Ergebnisse der Pilotierung deuten darauf hin, dass das Kodierverfahren zuverlässig ist (Cohen's Kappa: 0,7-0,96) und im beobachteten Unterricht der sechs Lehrpersonen Contingency bei bestimmten Lernaktivitäten (niedrige Autonomie) eher auftritt als bei anderen (hohe Autonomie).

Schlüsselwörter

Scaffolding · Adaptivität · Problemlösen . Autonomie $\cdot$ Umgang mit Heterogenität

\section{Measuring contingency during autonomous learning phases in physical education. Piloting of an observation instrument}

Abstract

Adaptive teaching is seen as a promising approach to deal with the great interindividual heterogeneity among students in physical education. The importance of the topic results on the one hand from the equality policy demand for individual support, but also from a learning and educational theory necessity to adapt learning environments to individual needs in order to support learning and educational processes. The fit between the teacher's instruction and the pupils' preconditions is a central quality criterion for dealing with heterogeneity. The research landscape on this topic is characterized by an enormous diversity in terms of the interpretation of and research methodological approach to adaptive teaching. This paper presents an approach for standardized observation of the contingency between instruction and student learning activities. Furthermore, results of a pilot study are presented. Contingency is operationalized as the relationship between instructions and previous learning activities. The results of the pilot study suggest that the coding method is reliable (Cohen's kappa $0.7-0.96$ ) and that contingency is more likely to occur in certain learning activities (low autonomy) than in others (high autonomy) in the observed instruction of the six teachers.

Keywords

Scaffolding · Adaptive teaching · Problem-solving · Autonomy · Dealing with heterogeneity richt als Contingency-Dimensionen rekonstruiert (Wibowo, 2015).

Für die Dimension der Autonomie spricht, dass diese als wichtiger Baustein eines theoretischen und empirischen Verständnisses eines zeitgemäßen Sportunterrichts ausgewiesen werden kann (Balz \& Neumann, 2007; Prohl, 2013;
Wibowo \& Dyson, 2020, im Review), in dem die angemessene Verteilung von Verantwortung für die Lernprozesse der Schüler*innen zwischen Lehrperson und Schüler*innen eine wichtige Rolle spielt (Prohl, 2010). Nicht zuletzt spricht für die Dimension der Autonomie, dass der Contingency-Approach 
aus der Forschung zum Scaffolding (Wood et al., 1978) eine theoretisch gut begründete und empirisch etablierte Grundlage zur Beurteilung von Contingency in dieser Dimension bietet. Weitergehend werden die Contingency-Einheiten für die Dimension der Autonomie aus Analyseeinheiten zur Autonomie der Schüler*innen und der Kontrolle der Lehrperson gebildet. Die Contingency-Regeln zur Relationierung von Autonomie und Kontrolle wurden in Anlehnung an den ContingencyApproach und der Annahme abgeleitet, dass solche Unterstützung lernförderlich ist, "which lay just one level above his [the child; JW] current level" (Wood et al., 1978, S. 133). So konnten jeder Autonomiestufe Stufen der Kontrolle zugeordnet werden, von denen angenommen werden kann, dass sie lernförderlich sind (vgl. Wibowo, 2015, 2016; s. Abschnitt zum methodischen Vorgehen zur konkreten Operationalisierung der Schüler*inneneinheiten, Lehrpersoneneinheiten und der ContingencyRegeln).

Für die Dimension des Problemlösens spricht, dass Problemlösen im Sportunterricht als fachspezifischer Ausdruck von Bildung und Lernen im Sportunterricht verstanden werden kann (Brodtmann \& Landau, 1982; Messmer, 2018, 2019; Prohl, 2013; Wibowo, 2015, 2019; Wright, MacDonald \& Burrows, 2004). Die Contingency-Einheiten werden demnach aus Problemlöseaktivitäten der Schüler*innen und der Aktivierung von Problemlöseaktivitäten der Lehrpersonen gebildet. Die ContingencyRegeln stützen sich auf die fachunspezifischen Annahmen, dass Aktivitäten der Lehrperson dann lernförderlich sind, wenn sie auf die (Problemlöse-)Aktivitäten der Schüler*innen eingehen (Analysieren der Schüler*innen, gefolgt von Aktivierung von Analysieren durch die Lehrperson; Fürst, 1999) oder wenn bei weiterführende Problemlöseaktivitäten angeregt werden (Analysieren der Schüler*innen gefolgt von Aktivierung von Planen durch die Lehrperson; vgl. zum Vorgehen Wibowo, 2015, 2019; s. Abschnitt zum methodischen Vorgehen zur konkreten Operationalisierung der Schüler*inneneinheiten, Lehrper- soneneinheiten und der ContingencyRegeln).

\section{Fragestellungen}

Aufgrund des oben dargestellten Forschungsstandes und der konzeptionellen Grundlagen wurden für die vorliegende Untersuchung folgende Fragestellungen formuliert:

1. Wie handeln Schüler*innen in selbstständigen Arbeitsphasen in Bezug auf die Autonomie und das Problemlösen?

2. Wie handeln Sportlehrpersonen in selbstständigen Arbeitsphasen in Bezug auf die Kontrolle und die Aktivierung von Problemlöseprozessen?

3. Wie ist das Verhältnis (Contingency) zwischen den Lernprozessen der Schüler*innen und der Instruktion der Lehrpersonen in den beiden Dimensionen Autonomie und Problemlösen?

\section{Methodisches Vorgehen}

\section{Stichprobe}

Für die Untersuchung wurde ein Videodatenpool der Frankfurter Studien zu Kooperativem Lernen im Sportunterricht reanalysiert (Bähr, Prohl, \& Gröben, 2008). Der Videodatenpool dokumentiert sechs 5. Klassen mit 159 Schüler*innen, die in einer sechswöchigen Unterrichtsreihe nach den kooperativen Lernformen Gruppenpuzzle und Gruppenturnier zu den Inhalten Handstand und Flugrolle unterrichtet wurden (Bähr \& Wibowo, 2018). In allen Klassen wurden die gleichen Unterrichtsmaterialien (Aufgabenkarten, Bildreihen, Sportgeräte) eingesetzt und die Lehrpersonen wurden instruiert, sich bei der Lernbegleitung eher zurückzuhalten und als Moderator*in zu agieren. Bei den Lehrpersonen handelte es sich um Freiwillige, die eine dreistündige Schulung erhalten haben (dies kann als vergleichsweise geringe Intervention eingestuft werden). Aus der Analyse der Implementationsgüte geht hervor, dass die Lehrpersonen sich hinsichtlich der Lernbegleitung nur eingeschränkt konzeptgemäß verhalten haben (Bähr, 2009). Es war daher vor der Durchführung dieser Studie zu erwarten, dass eine deutliche Streuung im Lehrpersonenhandeln zu beobachten sein würde und kein konzeptgemäßes, vorwiegend zurückhaltendes Handeln.

Bezüglich der Anzahl der zu erhebenden Messzeitpunkte werden in den gesichteten Studien unterschiedliche Strategien zwischen ein (Ayvazo \& Ward, 2011; Van de Pol et al., 2015; Wischgoll et al, 2015) bis fünf (Van de Pol et al., 2018) Messzeitpunkten in unterschiedlichen Unterrichtsstunden verfolgt. Aus forschungsökonomischen Gründen wurden vier Messzeitpunkte (unterschiedliche Unterrichtsstunden) aus den sechs im Datenpool vorhandenen Unterrichtsstunden pro Lehrperson ausgewählt. Aus diesen vier Unterrichtsstunden, wurden wiederum 20-minütige Sequenzen von Kleingruppenarbeit/Lernbegleitung ausgewählt (wie auch bei Wischgoll et al., 2015), in den die Schülerinnen über einen gewissen Spielraum für Entscheidungen verfügten (Bähr \& Wibowo, 2018). Die Fokussierung solcher Unterstützungsphasen findet sich auch bei Van de Pol et al. (2015) und Wischgoll et al. (2015). Durch Fokussierung solcher Arbeits-/ Unterstützungsphasen soll auf die in anderen Studien beobachtete Variabilität instruktionsbezogener Lehrpersonenhandlungen eingegangen werden (Kane \& Staiger, 2012; Praetorius et al., 2014). Praetorius et al. (2014) verweisen darauf, dass Instruktionsvariablen stark von Stunde zu Stunde variieren; dies ist auch für die Variablen der Lehrpersonen als Bestandteil der Contingency-Einheiten zu erwarten (s. unten). Die Autor*innen führen die zum Teil starke Varianz im Handeln der Lehrpersonen unter anderem auf unterschiedliche Schwerpunkte im Verlauf von Unterrichtsreihen (Einführung in ein Thema, Erarbeitung von Grundlagen, Prüfungsstunden) und innerhalb einzelner Stunden (durch unterschiedliche Unterrichtsphasen) zurück. Durch das Fokussieren ähnlicher Unterrichtsphasen sollten die Daten der einzelnen Unterrichtsstunden besser zueinander vergleichbar werden, und es werden gegenüber einer breiten Varianz an Unterrichtsphasen in kompletten Stunden weniger Messzeitpunkte nötig 
(Praetorious et al., 2014, empfehlen neun Messzeitpunkte für eine stabile Erfassung instruktionsbezogener Variablen).

\section{Datenerhebung}

Während in der Studie von Van de Pol et al. (2018) die Schüler ${ }^{*}$ innen-Lehrperson-Interaktionen ausschließlich über Audioaufnahmen erfasst werden, ist für den Bereich des Sportunterrichts die videographische Erfassung der Bewegungsaktivitäten (ggf. von Hilfestellungen oder Auf- bzw. Umbauprozessen) unerlässlich, da diese als Indikatoren im Event-Sampling-Verfahren (Döring \& Bortz, 2016) zur Einteilung der Schüler*innen bzw. Lehrpersoneinheiten verwendet werden (s. unten). In dem vorhandenen Datenpool konnte für alle Unterrichtsstunden auf eine Lehrpersonverfolgerkamera und eine Klassentotal-Perspektive zurückgegriffen werden. Die sprachliche Interaktion wurde über ein Funkmikrofon der Lehrperson erfasst. Die Videodaten wurden transkribiert und protokollartig um Deskriptionen relevanter Aktivitäten Bewegungs(versuche); Hilfestellungen ergänzt.

\section{Datenauswertung}

Die Kodierung der Adaptivität basiert wie bei anderen Studien (Ayvazo \& Ward, 2011; Hermkes et al., 2018; Van de Pol \& Elbers, 2013; Wischgoll et al., 2015) auf einem Dreischritt der Kodierung von Schüler*innenaktivitäten (hier: Autonomie, Problemlösen), Lehrpersonenaktivitäten (hier: Kontrolle, Aktivierung von Problemlöseaktivitäten) und der Contingency-Einheiten („contingent, non-contingent").

\section{Kodierung der Schüler*innen- und Lehrpersoneinheiten}

Die Analyseeinheiten für die Schülerinnenaktivitäten und Lehrpersonenaktivitäten wurden im Sinne eines Eventsamplings durch Sprechakt- oder Aktivitätswechsel festgelegt (Döring \& Bortz, 2016). Die Kodierung erfolgte für alle Analyseeinheiten durch zwei geschulte unabhängige Rater*innen. Die Güte der Kodierung wurde über den Cohen's
Kappa-Koeffizienten $(\kappa)$ ermittelt. Die Rater*innen wurden in sieben Sitzung á 90 min durch den Untersuchungsleiter geschult. Zur Ermittlung der Zuverlässigkeit der Rater*innenurteile wurde für einen Datensatz mit 824 Kodiereinheiten von dem Untersuchungsleiter und einem Mitarbeiter eine Masterkodierung erstellt. Die Rater*innen mussten die Zuverlässigkeit ihrer Urteile an der Kodierung dieses Datensatzes nachweisen. Die Kodierung des Hauptdatensatzes führten zu gleichen Teilen immer zwei Rater*innen unabhängig voneinander durch, Unstimmigkeiten wurden diskursiv überarbeitet. Alle vier von den Rater*innen kodierten Skalen wurden mit guten bis sehr guten Übereinstimmungswerten erfasst (Cohen's Kappa für die einzelnen Skalen und jede einzelne Rater*in: Kontrolle: 0,93/0,7; Autonomie: 0,87/0,77; Unterstützung des Problemlösens: 0,71/0,9; Problemlösen: 0,96/0,88).

In der Dimension Autonomie wurde das Handeln der Schüler*innen nach einer fünfstufigen Skala kodiert, deren einzelne Ausprägungen in Anlehnung an Wibowo $(2015,2016)$ (a) durch die (Selbst-)Regulation und die inhaltlichen Beiträge und (b) die vorangegangene Unterstützung der Lehrperson definiert wurden. Die Unterstützung der Lehrperson wurde durch die Skala Kontrolle mit folgenden Ausprägungen kodiert:

1. keine regulatorische/inhaltliche Unterstützung (niedrig),

2. Aktivierung/Regulation von Problemlöseaktivitäten ohne inhaltlichen Beitrag,

3. Aktivierung/Regulation von Problemlöseaktivitäten mit inhaltlichem Beitrag,

4. vollständiges Analysieren/Planen bzw. physisches Unterstützen einer Bewegung (hoch).

Darauf aufbauend wurden die Aktivitäten der Schüler*innen durch die Skala Autonomie mit folgenden Ausprägungen kodiert:

1. Problemlöseaktivität gelingt nicht (niedrig),

2. Problemlöseaktivität erfolgt nach Kontrolle 4,
3. Problemlöseaktivität erfolgt nach Kontrolle 3,

4. Problemlöseaktivität erfolgt nach Kontrolle 2,

5. Problemlöseaktivität erfolgt ohne vorangegangene Aktivierung der Lehrperson oder nach Kontrolle 1 der Lehrperson (hoch).

In Bezug auf die Contingency-Dimension Problemlösen wurden die Analyseeinheiten zum Schüler ${ }^{\star}$ innenhandeln in Anlehnung an Wibowo $(2015,2016$, 2019) danach kodiert, ob diese analysieren, planen oder probieren. Analysieren wird dabei als eine Aktivität mit Fokus auf zuvor durchgeführte Aktivitäten und das Verstehen von Problemen verstanden. Dagegen wurden solche Schüler*inneneinheiten als Planen kodiert, wenn der Fokus auf zukünftige Aktivitäten und das Lösen von Problemen lag. Als Probieren wurden solche Einheiten kodiert, in denen bewegungsbezogene Aktivitäten vorbereitet und durchgeführt wurden. Das Handeln der Lehrpersonen wurde danach eingestuft, ob die Schüler*innen zum Analysieren, Planen und Probieren aktiviert wurden (vgl. Wibowo, 2015, 2019).

\section{Kodierung der Contingency- Einheiten}

Die Contingency-Einheiten werden durch Schüler*innen-LehrpersonenKombinationseinheiten gebildet, d.h. es wird ermittelt, ob die Aktivierung einer Lehrperson $\mathrm{zu}$ einer vorausgegangenen Aktivität der Schüler*innen passt. Die Contingency-Einheiten wurden binär in „,contingent“ und „nichtcontingent" kodiert.

Contingency in der Dimension Problemlösen wurde nach zwei Aspekten kodiert. Zum einen geht aus den Nürnberger Studien zum Gruppenunterricht hervor, dass die Instruktion der Lehrpersonen dann als Störgrößen im Lernprozess der Schülerinnen wirkt, wenn sie nicht an den Aktivitäten der Schüler*innen ansetzt (Fürst, 1999; Haag, Fürst, \& Dann, 2000). Daraus wurde abgeleitet, dass die Instruktion der Lehrperson dann in Bezug auf die Dimension Problemlösen adaptiert ist, wenn sie an der Problemlöseaktivität ansetzt, mit der die Schü- 
Tab. 1 Contingency-Regeln für die Bestimmung von Contingency-Einheiten als "Contingent" (respektive wurden alle anderen Einheiten als "nicht-contingent" eingestuft)

Dimension Autonomie

Autonomie 1 gefolgt von Kontrolle 3/4 Autonomie 2 gefolgt von Kontrolle 3 Autonomie 3 gefolgt von Kontrolle 2/3 Autonomie 4 gefolgt von Kontrolle $1 / 2$ Autonomie 5 gefolgt von Kontrolle 1

Dimension Problemlösen

Analysieren gefolgt von Aktivierung von Analysieren/Aktivierung von Planen Planen gefolgt von Aktivierung Planen/ Aktivierung von Probieren

Probieren gefolgt von Aktivierung Probieren/Aktivierung Analysieren

ler*innen gerade beschäftigt sind, d.h. die dem Lehrpersonenhandeln vorausgegangen ist (z. B. Aktivierung von Analysieren folgt Analysieren). Zum anderen wird in der Problemlöseforschung angenommen, dass die genannten Aktivitäten prozesslogisch aufeinanderfolgen. Demnach geht Analysieren einem Planen voraus, dem wiederum ein Probieren folgt (Funke, 2006; Leutner, Fleischer, Wirth, Greiff, \& Funke, 2012). Dieser Prozesslogik folgend, wurden die Aktivitäten der Lehrpersonen auch dann als adaptiert eingestuft, wenn eine fortführende Problemlöseaktivität angeregt wurde (z.B. Aktivierung von Planen folgt Analysieren; - Tab. 1).

Contingency in der Dimension $\mathrm{Au}$ tonomie wurde in Anlehnung an den Contingency-Approach operationalisiert (Wood et al., 1978). Um die Annahme zu operationalisieren, dass es lernförderlich ist, wenn durch die Kontrolle von den Schüler*innen eine Stufe der Autonomie verlangt wird, die über ihrem letzten Autonomiestatus liegt, wurden die Skalen Autonomie und Kontrolle umgekehrt zueinander in Beziehung gesetzt. Das heißt, wenn die Schüler*inneneinheit in einem niedrigen Status von Autonomie kodiert wurde, wurde die Contingency-Einheit dann als "contingent" kodiert, wenn eine hohe Kontrolle erfolgte. Dies begründet sich darin, dass durch eine hohe Kontrolle den Schüler*innen nur ein Teil der Verantwortung übergeben wird und sie lediglich leicht über ihrer aktuellen Auto- nomiestufe gefordert werden (• Tab. 1). Die Zuteilung der Kontrollstufen zu den Autonomiestufen erfolgt in Anlehnung an Wibowo (2015).

Die Kodierung der ContingencyEinheiten erfolgte aus forschungsökonomischen Gründen automatisiert mit Hilfe des Query-Tools der Software Atlas.ti (Scientific Software Development GmbH, Berlin Deutschland; Friese, 2015). Das Query-Tool ist ein Werkzeug, um komplexe Suchanfragen in umfangreichen Datenpools durchzuführen. Zur Formulierung der Suchanfragen kann u.a. auf Näherungsoperatoren zurückgegriffen werden, die es ermöglichen, dass eine Neukodierung der gesuchten Kodekombination stattfindet (z.B. Autonomie 1 gefolgt von Kontrolle 3 oder 4 wird mit Contingency 3 neukodiert). Ein solches Verfahren reduziert die Kodierarbeit erheblich, da der dritte Kodierschritt nicht mehr durch humane Rater*innen durchgeführt werden muss.

\section{Durchgeführte Analysen}

Bezugspunkt der durchgeführten Analysen sind die Summen aller Kodiereinheiten der Lehrpersonen (1928) und der Schüler*innen (2172) über alle sechs Klassen und alle vier Stunden. Die Kodiereinheiten wurden immer in beiden Dimensionen kodiert, sodass für die deskriptive Einordnung prozentuale Angaben gemacht werden, die sich jeweils auf die Gesamtmenge der jeweiligen Kodiereinheiten beziehen.

\section{Ergebnisse}

\section{Schülerhandeln in selbstständigen} Arbeitsphasen (Fragestellung 1)

In der Skala Autonomie (AU) zeigte sich eine relativ geringe Streuung um den Mittelwert von 2,96 (SD: 0,2; Max: 3,35; Min: 2,7) auf der fünfstufigen Skala (•Tab. 2). Aufgrund der Verteilung der einzelnen Ausprägungen zeigte sich ein ambivalentes Bild des Handelns der Schüler*innen. Einerseits zeigten sie sich in ihren Beiträgen immer wieder überfordert (24,4\% Autonomie 1), andererseits gelang es ihnen nach Aufforderungen durch die Lehrperson, in etwas weniger als jedem zweiten Fall eigene inhaltliche Beiträge beizusteuern (Autonomie 4). Das geringe Auftreten der Ausprägung Autonomie 2 könnte dafürsprechen, die Ausprägungen Autonomie 2 und Autonomie 3 als inhaltliche unterstütztes Ausführen zusammenzufassen (•Tab. 2). In $45 \%$ aller Problemlöseaktivitäten (PL) beschäftigten sich die Schüler*innen damit herauszufinden, was in einer vorangegangenen Aktion passierte, sie versuchten das Problem zu verstehen (Analysieren). Dabei wurden $64,8 \%$ Beiträge mit geringer oder gar keiner Unterstützung ausgeführt (Autonomie 4 und 5), was darauf hinweist, dass die inhaltliche Ausführung dieser Analyseprozesse weitgehend in der Hand der Schüler*innen blieb. In 37,1\% der Fälle versuchten sie Lösungen für bestehende Probleme zu entwickeln bzw. Pläne für das weitere Vorgehen aufzustellen. Beim Planen zeigten sich die Schüler*innen in geringerem Maße autonom und wurden in $80,7 \%$ der Fälle in einem mittleren oder hohen Maße unterstützt bzw. waren sogar überfordert (Autonomie 1, 2 und 3). 17,9\% der Aktivitäten während der Lernbegleitung wurden darauf verwendet, eine Planung umzusetzen bzw. ein Problem durch praktisches Tun zu lösen. Bei dieser Problemlöseaktivität zeigten sich die Schüler*innen am kompetentesten.

\section{Lernbegleitung in selbstständigen Arbeitsphasen (Fragestellung 2)}

In Bezug auf die Kontrolle durch die Lehrperson zeigte sich in den Kodiereinheiten über alle sechs Lehrpersonen ein Wert von 2,65 auf der vierstufigen Skala mit einer mittleren Streuung (SD: 0,93). Weiterhin kann beobachtet werden, dass in $18,4 \%$ der Fälle eine Aktivierung zum Analysieren stattfand, in 57,1 \% der Fälle das Planen der Schüler*innen aktiviert wurde und in $24,5 \%$ der Fälle eine Aktivierung zum Lösungen von Problemen durch konkretes bewegungsbezogenes Tun erfolgte (•Tab.3). Bei der Aktivierung des Analysierens und des Probierens fand die Unterstützung hauptsächlich durch eine vorwiegende Aktivitätsregulation ohne inhaltliche Beiträge statt (Kontrolle 2), während bei der Akti- 
Tab. 2 Schüler*innenhandeln kodiert nach Problemlösen und Autonomie

\begin{tabular}{|c|c|c|c|c|c|c|c|c|}
\hline & & & \multicolumn{6}{|c|}{ Autonomie (AU) } \\
\hline & & & 1 & 2 & 3 & 4 & 5 & Gesamt \\
\hline \multirow{16}{*}{$\begin{array}{l}\text { Problem- } \\
\text { lösen (PL) }\end{array}$} & \multirow[t]{4}{*}{ Analysieren } & Anzahl & 217 & 21 & 75 & 547 & 29 & 889 \\
\hline & & $\%$ von PL & 24,4 & 2,4 & 8,4 & 61,5 & 3,3 & 100,0 \\
\hline & & $\%$ von $\mathrm{AU}$ & 41,6 & 19,3 & 21,4 & 65,9 & 17,8 & 45,0 \\
\hline & & $\%$ der Gesamt & 11,0 & 1,1 & 3,8 & 27,7 & 1,5 & 45,0 \\
\hline & \multirow[t]{4}{*}{ Planen } & Anzahl & 234 & 83 & 274 & 97 & 44 & 732 \\
\hline & & $\%$ von PL & 32,0 & 11,3 & 37,4 & 13,3 & 6,0 & 100,0 \\
\hline & & $\%$ von $\mathrm{AU}$ & 44,8 & 76,1 & 78,3 & 11,7 & 27,0 & 37,1 \\
\hline & & $\%$ der Gesamt & 11,9 & 4,2 & 13,9 & 4,9 & 2,2 & 37,1 \\
\hline & \multirow[t]{4}{*}{ Probieren } & Anzahl & 71 & 5 & 0 & 186 & 90 & 353 \\
\hline & & $\%$ von PL & 20,1 & 1,4 & 0,0 & 52,7 & 25,5 & 100,0 \\
\hline & & $\%$ von $\mathrm{AU}$ & 13,6 & 4,6 & 0,0 & 22,4 & 55,2 & 17,9 \\
\hline & & $\%$ der Gesamt & 3,6 & 0,3 & 0,0 & 9,4 & 4,6 & 17,9 \\
\hline & \multirow[t]{4}{*}{ Gesamt } & Anzahl & 522 & 109 & 350 & 830 & 163 & 1974 \\
\hline & & $\%$ von $\mathrm{PL}$ & 26,4 & 5,5 & 17,7 & 42,0 & 8,3 & 100,0 \\
\hline & & $\%$ von $\mathrm{AU}$ & 100,0 & 100,0 & 100,0 & 100,0 & 100,0 & 100,0 \\
\hline & & $\%$ der Gesamt & 26,4 & 5,5 & 17,7 & 42,0 & 8,3 & 100,0 \\
\hline
\end{tabular}

Tab. 3 Lehrpersonenaktivitäten kodiert nach Kontrolle und Aktivierung des Problemlösens (AdP)

\begin{tabular}{|c|c|c|c|c|c|c|c|}
\hline & & & \multicolumn{5}{|c|}{ Kontrolle } \\
\hline & & & $1^{a}$ & 2 & 3 & 4 & Gesamt \\
\hline \multirow{9}{*}{$\begin{array}{l}\text { Aktivierung } \\
\text { des Problem- } \\
\text { lösens (AdP) }\end{array}$} & \multirow{3}{*}{$\begin{array}{l}\text { Aktivierung } \\
\text { d. Analysie- } \\
\text { rens }\end{array}$} & Anzahl & - & 202 & 102 & 51 & 355 \\
\hline & & $\%$ von AdP & - & 56,9 & 28,7 & 14,4 & 100,0 \\
\hline & & \% Gesamt & - & 10,5 & 5,3 & 2,6 & 18,4 \\
\hline & \multirow{3}{*}{$\begin{array}{l}\text { Aktivierung } \\
\text { d. Planens }\end{array}$} & Anzahl & - & 91 & 639 & 370 & 1100 \\
\hline & & $\%$ von AdP & - & 8,3 & 58,1 & 33,6 & 100,0 \\
\hline & & $\%$ Gesamt & - & 4,7 & 33,1 & 19,2 & 57,1 \\
\hline & \multirow{3}{*}{$\begin{array}{l}\text { Aktivierung } \\
\text { d. Probierens }\end{array}$} & Anzahl & - & 437 & $0^{b}$ & 36 & 473 \\
\hline & & $\%$ von AdP & - & 92,4 & 0,0 & 7,6 & 100,0 \\
\hline & & \% Gesamt & - & 22,7 & 0,0 & 1,9 & 24,5 \\
\hline \multirow[t]{3}{*}{-} & \multirow[t]{3}{*}{ Gesamt } & Anzahl & $(244)$ & 730 & 741 & 457 & 1928 \\
\hline & & $\%$ von AdP & - & 37,9 & 38,4 & 23,7 & 100,0 \\
\hline & & \% Gesamt & - & 37,9 & 38,4 & 23,7 & 100,0 \\
\hline \multicolumn{8}{|c|}{$\begin{array}{l}{ }^{\text {aD }} \text { Da die Ausprägung Kontrolle } 1 \text { keiner Aktivierung der Problemlösenskategorie zugeordnet werden } \\
\text { kann, sind die } 244 \text { Fälle von Kontrolle } 1 \text { nicht in der Kreuztabellenstatistik enthalten. Daher weichen } \\
\text { auch die Spaltensummenprozente von den im Text stehenden Werten ab, da hier die Gesamtheit } \\
\text { von } 2173 \text { Fällen zugrunde gelegt wird } \\
\text { bHinweise zum konkreten Tun (Probieren) wurden immer als Hinweise zum Planen verstanden. } \\
\text { Daher ist die Anzahl in dieser Zelle und der entsprechenden Zelle in } \mathbf{0} \text { Tab. } 3 \text { gleich null }\end{array}$} \\
\hline
\end{tabular}

vierung des Planens inhaltliche Hinweise dominierten (Kontrolle 3).

\section{Contingency während selbstständiger Arbeitsphasen (Fragestellung 3)}

Bezogen auf die Dimension Autonomie wurden 50,1\% der Contingency-Einhei- ten als „contingent“ kodiert. Werden diese Fälle nach den einzelnen Stufen von Autonomie aufgeteilt, zeigt sich folgende Verteilung: 30,4\% der Fälle fanden bei einer Autonomie-1-Kodierung statt, 8,9\% der Fälle fanden bei einer Autonomie-2Kodierung statt, 26,2\% der Fälle fanden bei einer Autonomie-3-Kodierung statt, $31 \%$ fanden bei einer Autonomie-4-Ko- dierung statt und 3,5\% der Fälle fanden bei einer Autonomie-5-Kodierung statt.

In Bezug auf die Dimension Problemlösen wurden 60,9\% aller ContingencyEinheiten als „contingent“ kodiert. Diese verteilen sich wie folgt auf die einzelnen Problemlöseaktivitäten: „contingent“ bei Analysieren in 33,9\% der Fälle, , ,contingent" bei Planen in 46,6\% der Fälle und „contingent“ bei Probieren in 19,7\% der Fälle (•Tab.4).

\section{Diskussion}

\section{Einordnung der Ergebnisse}

Bezüglich der Reliabilität des pilotierten Beobachtungsverfahrens kann festgestellt werden, dass die ersten beiden Schritte (Kodierung von Autonomie, Problemlösen, Kontrolle und Aktivierung des Problemlösens) gut bis sehr gut gelingen. Auch der dritte Schritt der automatisierten Kodierung der Contingency-Einheiten mit Hilfe des QueryTools in Atlas.ti kann als hilfreich angesehen werden, da dieser Kodierschritt bei geeigneten Regeln mit vergleichsweise geringem Aufwand gegenüber der Kodierung durch humane Rater*innen erfolgen kann.

Die Ergebnisse der Pilotierung stellen aufgrund des Umfangs der Stichprobe 


\begin{tabular}{|c|c|c|c|c|c|c|c|c|}
\hline & & & \multicolumn{6}{|c|}{ Contingency in der Dimension Autonomie (CA) } \\
\hline & & & 1 & 2 & 3 & 4 & 5 & Gesamt \\
\hline \multirow{12}{*}{$\begin{array}{l}\text { Contingency } \\
\text { in der } \\
\text { Dimension } \\
\text { Problemlö- } \\
\text { sen (CP) }\end{array}$} & \multirow[t]{4}{*}{ Analysieren } & Anzahl & 127 & 16 & 77 & 74 & 1 & 295 \\
\hline & & $\%$ von $\mathrm{CP}$ & 43,1 & 5,4 & 26,1 & 25,1 & 0,3 & 100,0 \\
\hline & & $\%$ von $C A$ & 45,4 & 16,7 & 28,8 & 36,3 & 4,5 & 33,9 \\
\hline & & $\%$ Gesamt & 14,6 & 1,8 & 8,9 & 8,5 & 0,1 & 33,9 \\
\hline & \multirow[t]{4}{*}{ Planen } & Anzahl & 125 & 67 & 171 & 36 & 4 & 403 \\
\hline & & $\%$ von $\mathrm{CP}$ & 31,0 & 16,6 & 42,4 & 8,9 & 1,0 & 100,0 \\
\hline & & $\%$ von $C A$ & 44,6 & 69,8 & 64,0 & 17,6 & 18,2 & 46,4 \\
\hline & & $\%$ der Gesamt & 14,4 & 7,7 & 19,7 & 4,1 & 0,5 & 46,4 \\
\hline & \multirow[t]{4}{*}{ Probieren } & Anzahl & 28 & 13 & 19 & 94 & 17 & 171 \\
\hline & & $\%$ von $\mathrm{CP}$ & 16,4 & 7,6 & 11,1 & 55,0 & 9,9 & 100,0 \\
\hline & & $\%$ von CA & 10,0 & 13,5 & 7,1 & 46,1 & 77,3 & 19,7 \\
\hline & & $\%$ Gesamt & 3,2 & 1,5 & 2,2 & 10,8 & 2,0 & 19,7 \\
\hline \multirow[t]{4}{*}{ Gesamt } & & Anzahl & 280 & 96 & 267 & 204 & 22 & 869 \\
\hline & & $\%$ von $\mathrm{CP}$ & 32,2 & 11,0 & 30,7 & 23,5 & 2,5 & 100,0 \\
\hline & & $\%$ von CA & 100,0 & 100,0 & 100,0 & 100,0 & 100,0 & 100,0 \\
\hline & & $\%$ Gesamt & 32,2 & 11,0 & 30,7 & 23,5 & 2,5 & 100,0 \\
\hline
\end{tabular}

keine repräsentativen Ergebnisse dar, jedoch kann gezeigt werden, dass anhand des Instruments nicht nur Contingency im Sinne des Verhältnisses von Schülerinnenaktivität zu Lehrpersonenaktivität, sondern auch ein deutlich differenzierterer Blick auf die Aktivitäten der Schüler*innen und der Lehrkräfte erfolgen kann. Die Kreuzung der zwei Dimensionen für die jeweiligen Akteure hat das Potenzial, wertvolle Details über die Aktivitäten der jeweiligen Akteure zu gewinnen.

\section{Limitationen}

Die Studie unterliegt jedoch methodischen Limitationen. Aufgrund der Größe der Stichprobe der Lehrpersonen sind die Ergebnisse nur eingeschränkt für die Grundgesamtheit der Sportlehrer*innen generalisierbar. Die Generalisierung muss weiterhin durch die Themenfokussierung und die Fokussierung von 5. Klassen eingeschränkt werden. Durch größere Stichproben an Lehrpersonen mit einer größeren Streuung bezüglich der Unterrichtsthemen und Altersstufen könnten die Befunde (a) weiter generalisiert werden und (b) Gruppeneffekte (auf Schüler*innenmerkmale) ausgeschlos- sen werden, und es könnten (c) Aussagen zur Stabilität der Lehrpersonenmerkmale (über verschiedene Themen hinweg) getroffen werden. Weiterhin sollten für die Dimension des Problemlösens, die aus der Forschung zum Problemlösen abgeleiteten Contingency-Regeln validiert werden, da diese im Gegensatz zur Ableitung der Contingency-Regeln aus dem Contingency-Approach erstmalig eingesetzt wurden.

\section{Erfolge und Forschungs- perspektiven}

Durch das Einnehmen einer Contingency-Perspektive konnten in der Studie innovative Ergebnisse in der Forschung zur Lernbegleitung und zum Lernen im Sportunterricht hervorgebracht werden. In forschungsmethodischer Hinsicht wurde mit dem dreischrittigen Vorgehen zur Erfassung von Contingency ein aufwändiges, aber angemessenes Vorgehen vorgestellt, dass die Situationen der Schüler*innen detaillierter erfassen kann als vorangegangenen Studien. Gleiches gilt für die Lernbegleitung durch die Lehrpersonen in Bezug auf Kontrolle und Aktivierung des Problemlösens. Contingency als Kernmerkmal der Lern- begleitung und als einem Indikator für den Umgang mit Heterogenität kann im Rahmen dieser Studie damit präzise und verlässlich erfasst werden.

Jedoch besteht nach wie vor ein erheblicher Bedarf an Forschung zum Thema Adaptivität und Contingency im Sportunterricht. Lohnenswerte Perspektiven liegen u.a. in den Auswirkungen von Contingency auf die Nutzungsprozesse und Lernleistungen der Schüler*innen und Möglichkeiten, Contingency über die Fort- und Weiterbildung von Sportlehrpersonen zu beeinflussen. Beide Aspekte könnten aufgrund des noch überschaubaren Forschungsstandes von qualitativen, quantitativen oder auch Mixed-method-Ansätzen profitieren. Die fachspezifische Vertiefung des Kenntnisstandes zu Problemlöseprozessen könnte dabei nur eine gewinnbringende Fragestellung darstellen. Auf forschungsmethodischer Ebene besteht außerdem Bedarf, Stärken und Schwächen verschiedener Vorgehensweisen bei der Konstruktion von ContingencyEinheiten und Contingency-Regeln zu eruieren und (ggf. anhand von Urteilen von Expert*innen) zu validieren.

In Anschluss an die vorgestellte Pilotierung ist eine Hauptuntersuchung 
in Vorbereitung, in der Contingency als Indikator für die Performanz im Umgang mit Heterogenität verstanden wird und Zusammenhänge $\mathrm{zu}$ anderen Kompetenzfacetten zum Umgang mit Heterogenität (fachdidaktisches Wissen, Einstellungen zum Umgang mit $\mathrm{He}$ terogenität, professioneller Blick zum Umgang mit Heterogenität) untersucht werden sollen.

\section{Korrespondenzadresse}

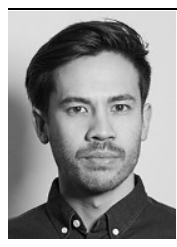

\section{Dr. Jonas Wibowo}

Bergische Universität

Wuppertal

Fuhlrottstr. 10, 42119 Wup-

pertal, Deutschland

Wibowo@uni-wuppertal.de

Funding. Open Access funding provided by Projekt DEAL.

\section{Einhaltung ethischer Richtlinien}

Interessenkonflikt. J. Wibowo gibt an, dass kein Interessenkonflikt besteht.

Für diesen Beitrag wurden von den Autoren keine Studien an Menschen oder Tieren durchgeführt. Für die aufgeführten Studien gelten die jeweils dort angegebenen ethischen Richtlinien.

Open Access Dieser Artikel wird unter der Creative Commons Namensnennung 4.0 International Lizenz veröffentlicht, welche die Nutzung, Vervielfältigung, Bearbeitung, Verbreitung und Wiedergabe in jeglichem Medium und Format erlaubt, sofern Sie den/die ursprünglichen Autor(en) und die Quelle ordnungsgemäß nennen, einen Link zur Creative Commons Lizenz beifügen und angeben, ob Änderungen vorgenommen wurden.

Die in diesem Artikel enthaltenen Bilder und sonstiges Drittmaterial unterliegen ebenfalls der genannten Creative Commons Lizenz, sofern sich aus der Abbildungslegende nichts anderes ergibt. Sofern das betreffende Material nicht unter der genannten Creative Commons Lizenz steht und die betreffende Handlung nicht nach gesetzlichen Vorschriften erlaubt ist, ist für die oben aufgeführten Weiterverwendungen des $\mathrm{Ma}$ terials die Einwilligung des jeweiligen Rechteinhabers einzuholen.

Weitere Details zur Lizenz entnehmen Sie bitte der Lizenzinformation auf http://creativecommons.org/ licenses/by/4.0/deed.de.

\section{Literatur}

Ayvazo, S., \& Ward, P. (2011). Pedagogical content knowledge of experienced teachers in physical education : functional analysis of adaptations. Research Quarterly for Exercise and Sport, 82(4), 675-684.
Bähr, I. (2009). Lehrer- und Schülerhandeln als methodologisches Problem bei der Implementierung von Unterrichtskonzepten. In H.P. BrandlBredenbeck (Hrsg.), Schulen in Bewegung Schulsport in Bewegung (S. 179-184). Hamburg: Czwalina Verlag.

Bähr, I., \& Wibowo, J. (2018). Theoretische Grundlagen - zum Verständnis Kooperativen Lernens. In J. Wibowo \& I. Bähr (Hrsg.), Kooperatives Lernen im Sportunterricht (S. 15-39). Baltmannsweiler: Schneider Verlag.

Bähr,I.,Prohl,R.,\&Gröben, B. (2008).Prozesse undEffekte "Kooperativen Lernens" im Sportunterricht. Unterrichtswissenschaft, 36(4), 290-308.

Balz, E., \& Neumann, P. (2007). Erziehender Sportunterricht. In R. Laging (Hrsg.), Neues Taschenbuch des Sportunterrichts (3. Aufl. S. 47-77). Baltmannsweiler: Schneider Verlag.

Beck, E., Baer, M., Guldimann, T., Bischoff, S., \& Brühwiler, C. (2008). Adaptive Lehrkompetenz. Münster:Waxmann.

Brodtmann, D., \& Landau, G. (1982). An Problemen lernen.sportpädagogik, 8(1), 16-22.

Corno, L. (2008). On teaching adaptively. Educational Psychologist, 43(3), 161-173.

Corno, L., \& Snow, R.E. (1986). Adapting teaching to individual differences among learners. In M. C. Wittrock (Hrsg.), Handbook of Research on Teaching (S.605-629). New York: Macmillan.

Decristan, J., Hondrich, A. L., Büttner, G., Hertel, S. Klieme, E., Kunter, M., et al. (2015). Impact of additional guidance in science education on primary students' conceptual understanding. The Journal of Educational Research, 108(5), 358-370.

Döring, N., \& Bortz, J. (2016). Forschungsmethoden und Evaluation in den Sozial- und Humanwissen schaften. Berlin, Heidelberg: Springer.

Farias, C., Hastie, P. A., \& Mesquita, I. (2018). Scaffolding student-coaches' instructional leadership toward student-centred peer interactions. European Physical Education Review, 24(3), 269-291. https://doi.org/10.1177/1356336X16687303.

Friese, S. Atlas.ti 7. User Guide and Reference, QUARC. http://downloads.atlasti.com/docs/manual/ atlasti_v7_manual_en.pdf?_ga=2.139200563. 1029542535.1495551950-1441297248. 1491805499. Zugegriffen: 21.09.2020.

Funke, J. (2006). Komplexes Problemlösen. In J. Funke (Hrsg.), Denken und Problemlösen (S. 375-445) Göttingen: Hogrefe Verlag für Psychologie.

Fürst, C. (1999). Die Rolle der Lehrperson im Gruppenunterricht. In H.-D. Dann, T. Diegritz \& H.S. Rosenbusch (Hrsg.), Gruppenunterricht im Schulalltag. Realität und Chancen (S. 107-150). Erlangen:Univ.-Bund Erlangen-Nürnberg..

Gräsel, C., Decristan, J., \& König, J. (2017). Adaptiver Umgang mit Heterogenität im Unterricht. Unterrichtswissenschaft, 45(4), 195-206.

Haag, L., Fürst, C., \& Dann, H.-D. (2000). Lehrervariablen erfolgreichen Gruppenunterrichts. Psychologie in Erziehung und Unterricht, 47(4), 266-279.

Hardy, I., Decristan, J., \& Klieme, E. (2019). Adaptive teaching in research on learning and instruction. Journal for educational research online, 11(2), 169-191.

Heemsoth, T., \& Wibowo, J. (2020). Fachdidaktisches Wissen von SportLehrpersonenn messen. German Journal of Exercise and Sport Research. https://doi.org/10.1007/s12662-020-00643-0.

Helmke, A., \& Weinert, F.E. (1997). Bedingungsfaktoren schulischer Leistungen. In F.E. Weinert (Hrsg.), Psychologie des Unterrichts und der Schu- le (S. 71-176). Göttingen: Hogrefe Verlag für Psychologie.

Hermkes, R., Mach, H., \& Minnameier, G. (2018). Interaction-based coding of scaffolding processes. Learning and Instruction, 54, 147-155. https:// doi.org/10.1016/j.learninstruc.2017.09.003.

Kane, T. J., \& Staiger, D. O. (2012). Gathering feedback for teachers: Combining high-quality observations with student surveys and achievement gains. Research Paper for the Bill \& Melinda Gates foundation.

Krammer, K. (2009). Individuelle Lernunterstützung in Schülerarbeitsphasen. Eine videobasierte Analyse des Unterstützungsverhaltens von Lehrpersonen im Mathematikunterricht. Münster:Waxmann.

Kunter, M., Klusmann, U., Baumert, J., Richter, D., Voss, T., \& Hachfeld, A. (2013). Professional competence of teachers: effects on instructional quality and student development. Journal of Educational Psychology, 105, 805-820.

Leutner, D., Fleischer, J., Wirth, J., Greiff, S., \& Funke, J. (2012). Analytische und dynamische Problemlösekompetenz im Lichte internationaler Schulleistungsvergleichsstudien. Untersuchungen zur Dimensionalität. Psychologische Rundschau, 63(1), 34-42.

Messmer, R. (2018). What is the subject matter of physical education? German Journal of Exercise and Sport Research, 48(4), 508-515. https://doi. org/10.1007/s12662-018-0531-2.

Messmer, R. (2019). Lernen (im Sport) sichtbar machen. In J. Wibowo, C. Krieger \& F. Bükers (Hrsg.), Aktivierung im Sportunterricht (S.52-62) Hamburg:Universität Hamburg.

Parsons, S. A., Vaughn, M., Scales, R. Q., Gallagher, M. A., Parsons, A. W., Davis, S. G., et al. (2018). Teachers' Instructional Adaptations: A Research Synthesis. Review of Educational Research, 88(2), 205-242.

Praetorius, A.-K., Pauli, C., Reusser, K., Rakoczy, K., \& Klieme, E. (2014). One lesson is all you need? Stability of instructional quality across lessons. Learning and Instruction, 31, 2-12. https://doi. org/10.1016/j.learninstruc.2013.12.002.

Prohl, R. (2010). Grundriss der Sportpädagogik (3. Aufl.). Wiebelsheim: Limpert.

Prohl, R. (2013). Sportpädagogik als Wissenschaftsdisziplin - Eine Standortbestimmung mit empirischem Ausblick. Zeitschrift für Sportpägogische Forschung, 1(1), 5-30.

Van de Pol, J., \& Elbers, E. (2013). Scaffolding student learning: A micro-analysis of teacherstudent interaction. Learning, Culture and Social Interaction, 2, 32-41.

Van de Pol, J., Mercer, N., \& Volman, M. (2018). Scaffolding student understanding in smallgroup work. Students' uptake of teacher support in subsequent small-group interaction. Journal of the Learning Sciences, 28(2), 206-239. https:// doi.org/10.1080/10508406.2018.1522258.

Van de Pol, J., Volman, M., \& Beishuizen, J. (2010). Scaffolding in teacher-student interaction: a decade of research. Educational Psychology Review, 22(3),271-296. https://doi.org/10.1007/ s10648-010-9127-6.

Van de Pol, J., Volman, M., Elbers, E., \& Beishuizen, J. (2012). Measuring Scaffolding in Teacher-Small-Group Interactions. In R. M. Gillies (Hrsg.), Pedagogy. New developments in the learning sciences (S. 151-188). Hauppauge: Nova Science Publishers.

Van de Pol, J., Volman, M., Oort, F., \& Beishuizen, J. (2015). The effects of scaffolding in the classroom: support contingency and student independent working time in relation to student 
achievement, task effort and appreciation of support. Instructional Science, 43, 615-641. https://doi.org/10.1007/s11251-015-9351-z.

Vogler, J., Messmer, R. \& Allemann, D. (2017). Das fachdidaktische Wissen und Können von Sportlehrpersonen (PCK-Sport). German Journal of Exercise and Sport Research, 47(4), 335-347. https://doi.org/10.1007/s12662-017-0461-4.

Wibowo, J. (2015). Betreuung selbständigen Lernens im Sportunterricht. Hamburg:Universität Hamburg.

Wibowo, J. (2016). Adaptives Lehrerhandeln im Sportunterricht. Zeitschrift für Sportpädagogische Forschung (Sonderheft 1), 63-84.

Wibowo, J. (2019). Zum Verhältnis von Aktivität, Aktivierung und Adaption am Beispiel von Problemlöseprozessen im Sportunterricht. In J. Wibowo, C. Krieger \& F. Bükers (Hrsg.), Aktivierung im Sportunterricht (S. 90-103). Hamburg: Universität Hamburg.

Wibowo,J.\&Dyson, B. (2020, im Review). A contingency perspective on learning and instruction in Physical Education. European Physical Education Review.

Wibowo, J. \& Heemsoth, T. (2019). Fachdidaktisches Wissen von Sportlehrer*innen testen: Überlegungen zur Inhaltsvalidität. Zeitschrift für Sportpädagogische Forschung, 7(2), 88-108.

Wibowo, J., Bähr, I.\& Gröben, B. (2014). Scaffolding as an Instruction model for the Cooperative Learning model in Physical Education. Active and Healthy Magazine, 21(2), 15-18.

Wischgoll, A., Pauli, C., \& Reusser, K. (2015). Scaffolding-How can contingency lead to successful learning when dealing with errors? ZDM Mathematics Education, 47(7), 1147-1159. https://doi. org/10.1007/s11858-015-0714-3.

Wood, D., Bruner, J.S., \& Ross, G. (1976). The role of tutoring in problem solving. Journal of child psychology and psychiatry, 17(17), 89-100. https:// doi.org/10.1111/j.1469-7610.1976.tb00381.x/ pdf.

Wood, D., Wood, H., \& Middleton, D. (1978). An experimental evaluation of four face-to-face teaching strategies. International Journal of Behavioral Development, 1(2), 131-147. https:// doi.org/10.1177/016502547800100203.

Wright, J., MacDonald, D., \& Burrows, L. (Hrsg.). (2004). Critical inquiry and problem-solving in physical education. London: Routledge. 